\title{
Caracterização física de agregados do solo submetido a 10 anos de aplicação de dejetos suínos
}

\author{
Physical characterization of soil aggregates submitted to 10 years-old of application of \\ swine manure
}

\section{Carlos Aristeu Mergen Junior ${ }^{1}$; Arcângelo Loss²; Elano Dos Santos Junior ${ }^{3}$; Lucas Dupont Giumbelli; ; Daniela de Pinho5; Lucinéia de Abreu'; Cledimar Rogério Lourenzi"; Jucinei José Comin ${ }^{8}$; Gustavo Brunetto ${ }^{9}$}

\footnotetext{
${ }^{1}$ MSc. Universidade Federal de Santa Catarina, Santa Catarina, Brasil, carlosmergenjunior@gmail.com.

${ }^{2}$ Professor, Ph.D. Universidade Federal de Santa Catarina, Santa Catarina, Brasil, arcangelo.loss@ufsc.br.

${ }^{3}$ I.A. Universidade Federal de Santa Catarina, Santa Catarina, Brasil, elanoagro@gmail.com.

${ }^{4}$ I.A. Universidade Federal de Santa Catarina, Santa Catarina, Brasil, lukdg@hotmail.com.

${ }^{5}$ I.A. Universidade Federal de Santa Catarina, Santa Catarina, Brasil, daniela_pnh@hotmail.com.

${ }^{6}$ I.A. Universidade Federal de Santa Catarina, Santa Catarina, Brasil, lucideabreu@gmail.com.

${ }^{7}$ Professor. Ph.D. Universidade Federal de Santa Catarina, Santa Catarina, Brasil, lourenzi.c.r@ufsc.br.

${ }^{8}$ Professor. Ph.D. Universidade Federal de Santa Catarina, Santa Catarina, Brasil, j.comin@ufsc.br.

${ }^{9}$ Professor. Ph.D. Universidade Federal de Santa Maria, Santa Maria, Brasil, brunetto.gustavo@gmail.com.
}

Citação: Mergen, C., Loss, A., Dos Santos, E., Dupont, L., Pinho, D., De Abreu, L., Lourenzi, C., Comin, J. \& Brunetto, G. (2019). Caracterização física de agregados do solo submetido a 10 anos de aplicação de dejetos suínos. Revista de Ciencias Agrícolas. 36(1): 79-92. doi: http://dx.doi.org/10.22267/rcia.193601.100

Recebido: 16 de novembro de $2018 . \quad$ Aprovado: 21 de fevereiro de 2019.

\section{RESUMO}

Aplicações sucessivas de dejetos suínos podem promover mudanças nos atributos físicos do solo, com ênfase nas vias de formação dos agregados solo. 0 objetivo deste trabalho foi separar e quantificar os agregados conforme sua via de formação e avaliar a estabilidade desses agregados em área com histórico de aplicações de dejeto líquido de suínos (DLS) e cama sobreposta de suínos (CSS), em sistema plantio 
direto (SPD). Após 10 anos do uso de dejetos suínos (DS) na sucessão aveia/milho sob SPD, coletaram-se amostras indeformadas de solo nas camadas de 0-5 e 5-10 cm, nos tratamentos sem DS (testemunha), com aplicação de DLS e CSS em doses equivalentes a uma e duas vezes a recomendação de N para o milho e a aveia (DLS1X, DLS2X, CSS1X e CSS2X, respectivamente). Os agregados foram separados conforme os seus padrões morfológicos e vias de formação em biogênicos e fisiogênicos. Em seguida, avaliaramse o diâmetro médio ponderado (DMP) dos agregados e a distribuição dos agregados nas classes de diâmetro para macroagregados $(8,00>\emptyset \geq 2,0 \mathrm{~mm})$, mesoagregados $(2,0>\varnothing \geq 0,25 \mathrm{~mm})$ e microagregados $(\varnothing<0,25 \mathrm{~mm})$, em cada tipo de agregado. A adição de dejetos suínos aumentou a formação de agregados biogênicos. Na área testemunha prevalece a via de formação de agregados fisiogênicos. A aplicação de DLS não alterou o DMP e os macroagregados em relação à testemunha, enquanto a aplicação de CSS aumentou esses parâmetros quando comparada aos tratamentos DLS e Testemunha.

Palavras chave: Agregados biogênicos, agregados fisiogênicos, cama sobreposta de suínos, dejeto líquido de suínos, índices de agregação.

\begin{abstract}
Successive applications of swine manure can promote changes in soil physical attributes, with emphasis on the soil aggregation pathways. The objective of this work was to separate and quantify the aggregates according to their training path and to evaluate the stability of these aggregates in an area with a history of applications of pig slurry (PS) and deep litter (DL) in no-tillage system (NTS). After 10 years of use swine manure (SM) in oat/corn succession under NTS, undisturbed soil samples were collected in the 0-5 and 5-10 cm layers, in the treatments without SM (control), with application of PS and DL at doses equivalent to one and two times the recommended N for corn and oats (PS1X, PS2X, DL1X and DL2X, respectively). The aggregates were separated according to their morphological patterns and biogenic and physicogenic formation pathways. Then, weighted average diameter (WMD) of the aggregates and the distribution of the aggregates in the diameter classes for macroaggregates $(8.0>\emptyset \geq 2.0 \mathrm{~mm})$, mesoaggregates $(2.0>\emptyset \geq 0.25 \mathrm{~mm})$ and microaggregates $(\varnothing<0.25 \mathrm{~mm})$ in each type of aggregate were evaluated. The addition of SM increased the formation of biogenic aggregates. In the control area, the formation pathway of physicogenic aggregates prevails. The application of PS did not alter the WMD and the macroaggregates in relation to the control, while the application of DL increased these parameters when compared to the PS and control treatments.
\end{abstract}

Keywords: Biogenic aggregates, physicogenic aggregates, deep litter, pig slurry, aggregation indices.

\section{INTRODUÇÃO}

Em Santa Catarina tem-se um grande número de animais de exploração zootécnica, com destaque para a criação de suínos, a qual apresentou um rebanho de 10,7 milhões de cabeças no ano de 2016 (IBGE, 2016). Nesses sistemas de produção, são gerados enormes volumes de dejetos, sendo que uma opção de aproveitamento destes é o seu uso como adubo na fertilização do solo, pois possuem nutrientes essenciais em sua composição e potencial de aumentar o rendimento dos cultivos agrícolas (Giacomini \& Aita, 2008). Contudo, o uso de dejetos suínos como adubos não é exclusivamente benéfico, havendo a necessidade de se comporem critérios, a exemplo do acúmulo de nutrientes em quantidades indesejáveis no solo e sua passagem para os mananciais (Seganfredo, 2007). Uma maneira muito comum de descarte desses dejetos nas propriedades agrícolas é a sua aplicação no solo, quase sempre sem critérios técnicos e de maneira periódica. Dessa maneira, é premente a necessidade de estudos 
que visem compreender de maneira satisfatória a dinâmica do uso de dejetos na adubação das lavouras em relação aos atributos do solo.

Em condições naturais, as partículas primárias do solo (areia, silte e argila) agrupam-se em unidades compostas por meio dos processos físico-químicos (floculação e expansão e contração das massas de argilas) e biológicos (atividade dos organismos e influência da matéria orgânica do solo), sendo então chamadas de agregados do solo, e este fenômeno é conhecido como agregação (Tivet et al., 2013). A presença de agregados no solo é um componente de estudo fundamental, pois confere ao solo diversas propriedades benéficas. Para Vezzani \& Mielniczuck (2011), a agregação é uma propriedade emergente do solo, quando este é encarado como sistema, pois é um processo que cresce em complexidade formando agregados maiores até o estágio de conferir características que permitem a otimização de seu funcionamento, mais precisamente no contexto do solo exercer seu papel no meio ambiente.

A agregação exerce efeito principalmente nas propriedades do solo relativas à aeração, permeabilidade, taxas de infiltração e retenção de água no solo (Kampf \& Curi, 2012), mas também em outras propriedades relacionadas ao sequestro de carbono (Vezzani \& Mielniczuck, 2011; Loss et al., 2014) e retenção de nutrientes (Silva et al., 2010), por exemplo.

Os agregados do solo podem ser diferenciados de acordo com a sua gênese e, ou, vias de formação, em fisiogênicos e biogênicos, conforme padrões morfológicos (Velasquez et al., 2007; Jouquet et al., 2009; Loss et al., 2014; Loss et al., 2017). Os agregados fisiogênicos são aqueles que apresentam formas angulares ou prismáticas e estão relacionados com o processo de aproximação das partículas unitárias do solo relativas aos ciclos de umedecimento e secagem e pela ação de agentes cimentantes do solo (matéria orgânica e, ou, óxidos e hidróxidos de ferro e alumínio), e os agregados biogênicos são aqueles que apresentam formas arredondadas e estão relacionados às vias biológicas de formação, tais como a passagem pelo trato intestinal da macrofauna do solo, especialmente minhocas, e também à ação cimentante de exsudatos das raízes e da biota associada (Velasquez et al., 2007; Batista et al., 2013; Loss et al., 2014). Os agregados biogênicos, em função de serem excretas da macrofauna que se alimenta basicamente de matéria orgânica, apresentam maiores teores de nutrientes, contribuem na proteção física da matéria orgânica (sequestro de carbono), e normalmente possuem maior estabilidade física em água (Silva et al., 2010; Silva et al., 2016), o que pode incluílos como potenciais indicadores de qualidade do solo, uma vez que são sensíveis aos diferentes sistemas de uso e manejo do solo (Loss et al., 2014; Fernandes et al., 2017).

Em relação ao uso da adubação orgânica, aplicações continuadas de dejetos animais ao solo podem promover alterações na agregação do solo (Yague et al., 2012; Comin et al., 2013). Isto é decorrente da entrada de material orgânico, que favorece o processo de agregação, melhorando as características físicas do solo, especialmente formando agregados maiores que 2,00 mm, que são considerados indicadores de qualidade do solo (Vezzani \& Mielniczuck, 2011) e formando agregados mais estáveis em água (Yague et al., 2012). Em estudos de longa duração e especialmente nos tratamentos com cama sobreposta de suínos encontraram-se maiores proporções de macroagregados (Comin et al., 2013).

Sendo assim, estudos sobre a gênese de agregados classificados como biogênicos e fisiogênicos ainda são incipientes, e a caracterização desses agregados em relação aos índices de agregação podem contribuir para melhor compreensão da dinâmica do uso 
de dejetos em solos, identificando assim as melhores estratégias de manejo do solo para melhorar seus atributos físicos. A hipótese deste estudo é de que o uso de dejetos suínos por longo prazo aumenta a formação de agregados biogênicos em comparação aos fisiogênicos. 0 objetivo deste trabalho foi separar e quantificar os agregados conforme sua via de formação e avaliar a estabilidade desses agregados em área com histórico de aplicações de dejeto líquido de suínos e cama sobreposta de suínos, em sistema plantio direto.

\section{MATERIAIS E MÉTODOS}

\section{Caracterização da área de estudo e dos} tratamentos avaliados. 0 experimento foi instalado no ano de 2002, em um Argissolo Vermelho-Amarelo (EMBRAPA, 2013) cultivado sob sistema plantio direto (SPD) com a sucessão aveia (Avena strigosa) / milho (Zea mays), em uma propriedade rural localizada em Braço do Norte, SC. 0 clima da região é do tipo Cfa (subtropical úmido), com temperaturas médias anuais de $18,7^{\circ} \mathrm{C}$ e precipitação média anual de $1.471 \mathrm{~mm}$. Previamente à instalação do experimento, na camada de $0-10 \mathrm{~cm}$, o solo apresentava os seguintes parâmetros: $\mathrm{pH}-\mathrm{H}_{2} \mathrm{O}$ 5,1; argila $330 \mathrm{~g} \cdot \mathrm{kg}^{-1}$; Ca, $\mathrm{Mg}$ e Al trocáveis 3,0, 0,8 e $0,8 \mathrm{cmol}_{\mathrm{c}} \mathrm{dm}^{-3}$, respectivamente (extraídos por $\mathrm{KCl} 1 \mathrm{~mol} \cdot \mathrm{L}^{-1}$ ); P e K disponíveis 19 e 130 $\mathrm{mg} \cdot \mathrm{dm}^{-3}$, respectivamente (extraídos por Mehlich-1), matéria orgânica 33,0 $\mathrm{g} \cdot \mathrm{kg}^{-1}$.

Anteriormente a instalação do experimento, a área estava coberta por uma pastagem predominantemente de Paspalum sp., sendo esporadicamente feita aplicação de dejetos suínos. Em dezembro de 2002 foi aplicado, na superfície do solo, $6 \mathrm{Mg}^{-1}$ de calcário (PRNT $=87,5 \%)$, para elevar o pH em água até 6,0 (CFS-RS/SC, 1995). Em seguida, foram instalados os tratamentos: testemunha (sem adubação); adubação com dejeto líquido de suínos, equivalente a recomendação de $\mathrm{N}$ ha $^{-1}$ ano $^{-1}$ para a cultura do milho e da aveia (DLS1X); adubação com DLS, equivalente ao dobro da recomendação de $\mathrm{N} \cdot \mathrm{ha}^{-1} \cdot$ ano $^{-1}$ para a cultura do milho e da aveia (DLS2X); adubação com cama sobreposta de suínos, equivalente a recomendação de $\mathrm{N} \cdot \mathrm{ha}^{-1} \mathrm{ano}^{-1}$ para a cultura do milho e da aveia (CSS1X) e adubação CSS, equivalente ao dobro da recomendação de $\mathrm{N} \cdot \mathrm{ha}^{-}$ ${ }^{1}$. ano $^{-1}$ para a cultura do milho e da aveia preta (CSS2X).

0 delineamento experimental utilizado foi o de blocos casualizados, com cinco tratamentos e três repetições. Cada um dos blocos possuía dimensões de 4,5 x 42,0m, separados entre si por um corredor de um metro de largura, sendo as parcelas distanciadas entre si por corredores de $3 \mathrm{~m}$. Cada tratamento era formado por unidades experimentais (parcelas) com 4,5 x 6,0m $\left(27 \mathrm{~m}^{2}\right)$. Os DLS foram coletados em esterqueira de sistema de criação ciclo completo situada na mesma propriedade na qual o experimento foi instalado. A CSS foi obtida na Escola Agrotécnica Federal de Concórdia, onde o sistema de criação de suínos é feito com substrato de maravalha.

A quantidade necessária de dejetos para suprir a demanda de $\mathrm{N}$ para a sucessão milho/ aveia em cada tratamento, usada ao longo do período de 2002 até 2012 foi estabelecida de acordo com a recomendação proposta pela Comissão de Química e Fertilidade do Solo (CFS RS/SC, 1995, CQFS RS/SC, 2004). Assim, a quantidade de DLS1X e DLS2X a ser aplicada foi definida pela estimativa da matéria seca (MS) e da concentração de nutrientes no dejeto. Já a quantidade de CSS1X e CSS2X aplicada foi calculada com base na mineralização de $50 \%$ do $\mathrm{N}$ amoniacal contido no resíduo. A média de MS e volume de dejetos aplicados, assim como a caracterização dos macronutirentes dos dejetos ao longo dos 10 anos de aplicações encontramse na Tabela 1. 
Tabela 1. Volume de dejetos líquidos e quantidade de cama sobreposta de suínos aplicados no solo, e caracterização química dos dejetos durante o período experimental.

\begin{tabular}{|c|c|c|c|c|c|c|c|c|c|c|}
\hline \multirow{2}{*}{$\begin{array}{c}\text { Ano } \\
\text { agrícola }\end{array}$} & MS & $\mathrm{C} / \mathrm{N}$ & pH & CE & VA & Ca & Mg & $\mathrm{N}$ & $\mathbf{P}$ & $\mathbf{K}$ \\
\hline & $\%$ & --- & --. & ds $\mathbf{m}^{-1}$ & --- & \multicolumn{5}{|c|}{ 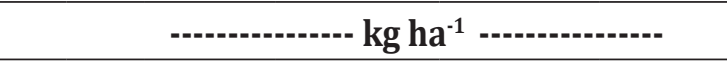 } \\
\hline \multirow[t]{2}{*}{$2002 / 12$} & \multicolumn{10}{|c|}{ Dejetos líquidos de suínos (DLS) } \\
\hline & 2,25 & 4,4 & 8,1 & 9,3 & 538,90 & 68,75 & 26,38 & 126,25 & 37,88 & 62,75 \\
\hline $2002 / 12$ & \multicolumn{10}{|c|}{ Cama sobreposta de suínos (CSS) } \\
\hline & 50,95 & 13,2 & 8,8 & 5,9 & 153,10 & 322,38 & 97,88 & 171,06 & 103,13 & 169,50 \\
\hline
\end{tabular}

MS = Massa Seca CE = Condutividade Elétrica; VA = Volume Aplicado, sendo para DLS em m ha-1 e para CSS em Mg ha ${ }^{-1}$. Para os dejetos, tem-se o somatório das quantidades totais aplicadas ao longo do período de 2002-2012. Para os macronutrientes tem-se a média dos valores obtidos ao longo de 2002-2012.

Afora o calcário na implantação, os DLS e a CSS foram as únicas fontes de insumos adicionadas na superfície do solo em SPD para a sucessão aveia/milho ao longo do período experimental, que foi de 2002 até 2012. As doses de DLS, em cada ano agrícola, foram aplicadas na superfície do solo e parceladas em quatro vezes, totalizando 40 aplicações de DLS (10 anos de experimentação e 4 parcelamentos), a saber: a $1^{\underline{a}}$ aplicação foi realizada na semana da semeadura do milho; a $2^{\mathrm{a}}$, aos 51 dias após a semeadura (DAS) do milho; a 3a , aos 95 DAS do milho e a 4aa aos 15 DAS da aveia. Para a CSS, foram realizadas dez aplicações durante o período experimental, sendo cada aplicação realizada na superfície do solo, em média, 15 a 30 dias antes da implantação de cada cultivo do milho (época do verão). Nos ciclos da aveia preta não foi realizada aplicação de CSS.

Em relação ao rendimento das culturas utilizadas no experimento, tem-se a produção média de matéria seca da aveia e da produção de grãos de milho durante o período de dez anos de aplicação dos dejetos, conforme Loss et al. (2017) (Tabela 2).
Tabela 2. Produção média por safra de matéria seca (MS) de aveia e grãos de milho, em Mg ha' ${ }^{-1}$, ao longo de 10 anos de aplicação de dejetos de suínos.

\begin{tabular}{lcc}
\hline \multicolumn{1}{c}{ Tratamentos } & MS da aveia & Milho \\
\hline Testemunha & 6,5 & 2,9 \\
DLS1X & 8,1 & 5,0 \\
DLS2X & 8,7 & 5,5 \\
CSS1X & 8,2 & 5,6 \\
CSS2X & 8,9 & 6,0 \\
\hline
\end{tabular}

Fonte: Loss et al. (2017). Testemunha (sem adubação com dejetos); DLS1X = dejeto líquido de suínos, 1 vez a quantidade; DLS2X = dejeto líquido de suínos, 2 vezes a quantidade; $\operatorname{CSS1X}=$ cama sobreposta de suínos, 1 vez a quantidade; $\operatorname{CSS} 2 \mathrm{X}$ = cama sobreposta de suínos, 2 vezes a quantidade.

Coleta de solo e análises realizadas. Em fevereiro de 2013, em cada tratamento, nas entrelinhas do milho abriu-se uma trincheira de 40x40x40 cm e com auxílio de uma pá reta e de uma espátula foram coletadas amostras indeformadas de solo nas camadas de 0-5 e $5-10 \mathrm{~cm}$ de profundidade. No laboratório, as amostras foram secas à sombra, sendo em seguida separadas conforme suas linhas de fraqueza e peneiradas em um conjunto de peneiras de malha de 9,5; 8,0 e 4,0mm, para obtenção dos agregados, conforme adaptado 
de EMBRAPA (1997). Após a separação dos agregados, obtiveram-se três amostras compostas por tratamento avaliado, sendo cada amostra composta formada por duas amostras indeformadas simples coletadas em cada unidade experimental. Para a separação dos agregados, conforme a via de formação, utilizaram-se os agregados contidos no intervalo de 9,5 a 4,0mm. Estes foram observados sob microscópico binocular e separados à mão conforme Velasquez et al. (2007). A separação dos agregados foi feita através de padrões morfológicos, sendo os agregados fisiogênicos, definidos por apresentarem formas angulares e subangulares (Figura 1a); e os agregados biogênicos, aqueles onde é possível a visualização de formas arredondadas, providas do trato intestinal dos indivíduos da macrofauna do solo e, ou, aqueles associados a atividade de raízes (Figura 1b).
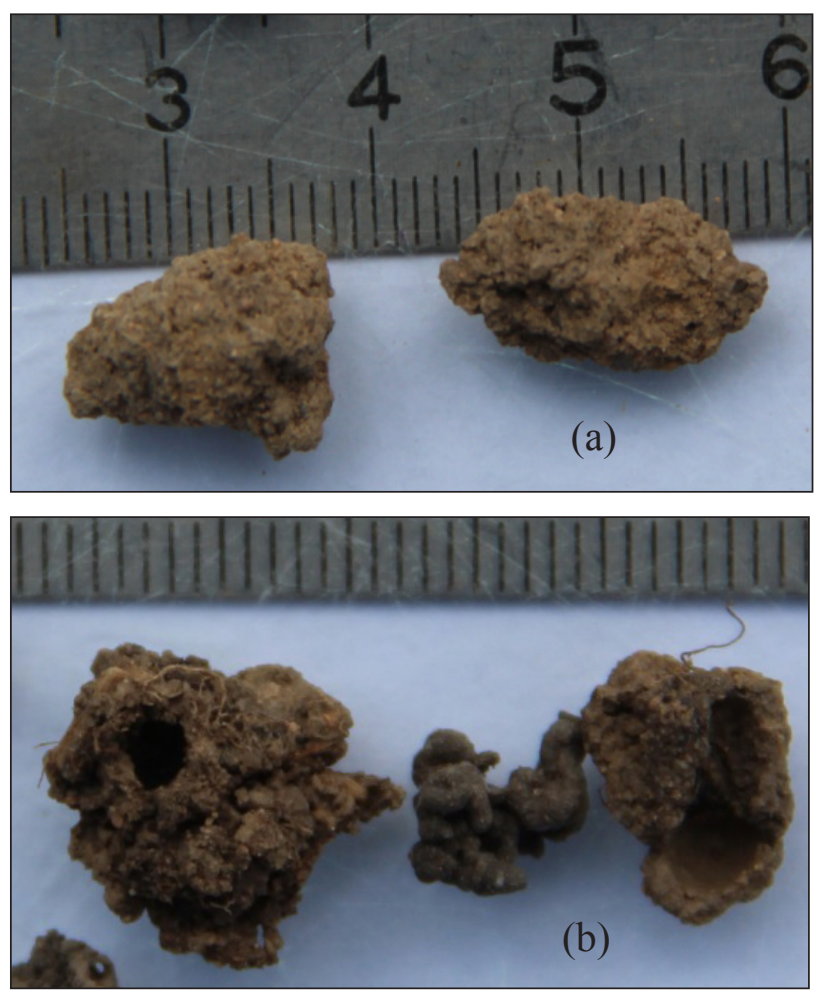

Figura 1. Agregados fisiogênicos (a) e biogênicos (b) de um Argissolo Vermelho-Amarelo após aplicações de dejetos líquidos e cama sobreposta de suínos em Braço do Norte, SC.
Para determinar a contribuição relativa dos agregados em termos de massa, pesaram-se todos os agregados biogênicos e fisiogênicos que foram identificados sob lupa e, assim, quantificado a fração de agregados fisiogênicos e biogênicos em relação à massa inicial. Após a separação em cada tipo de agregados procedeuse à análise de estabilidade de agregados.

Os agregados biogênicos e fisogênicos foram passados por peneira de 8,00 e $4,00 \mathrm{~mm}$. Em seguida foram pesados $25 \mathrm{~g}$ de agregados, dentre os retidos na peneira de $4,00 \mathrm{~mm}$, que foram transferidos para peneira de $2,00 \mathrm{~mm}$, assim compondo um conjunto de peneiras com diâmetro de malha decrescente, a saber: 2,$00 ; 1,00 ; 0,50 ; 0,25$ e $0,105 \mathrm{~mm}$. Os agregados inicialmente colocados na peneira de 2,00 $\mathrm{mm}$ foram umedecidos com borrifador e, posteriormente, o conjunto de peneiras foi submetido à tamisação vertical via úmida por 15 minutos no aparelho de Yoder (Embrapa, 1997). Transcorrido esse tempo, o material retido em cada peneira foi retirado, separado com jato d'água, colocado em placas de petri previamente pesadas e identificadas, e levado à estufa até a obtenção de massa constante. A partir da massa de agregados foram calculados o diâmetro médio ponderado (DMP) dos agregados, segundo Embrapa (1997). Ainda, com a massa dos agregados foi avaliada a sua distribuição nas seguintes classes de diâmetro médio: 8,00 $>\varnothing \geq 2,0 \mathrm{~mm}$ (macroagregados); $2,0>\emptyset \geq 0,25 \mathrm{~mm}$ (mesoagregados) e $\emptyset<0,25 \mathrm{~mm}$ (microagregados).

Análises estatísticas. Os resultados foram analisados quanto à normalidade e homogeneidade dos dados por meio dos testes de Lilliefors e Barttlet, respectivamente. Posteriormente, foi analisado como delineamento em blocos casualizados, com cinco tratamentos (Testemunha, DLS1X, DLS2X, CSS1X, CSS2X) e 3 repetições cada. Os tratamentos avaliados estão sob as mesmas condições topográficas e edafoclimáticas, diferindo apenas no tipo 
de adubação recebida. Os resultados foram submetidos à análise de variância com aplicação do teste $\mathrm{F}$ e os valores médios, quando significativos, comparados entre si pelo teste Scott-knott a 5\%, através do software Sivar 5.6.

\section{RESULTADOS E DISCUSSÃO}

\section{Gênese de Agregados Biogênicos e} Fisiogênicos. Verificaram-se maiores proporções de agregados biogênicos nas áreas com adição de dejetos suínos em comparação a área testemunha, para as duas profundidades avaliadas. Na área testemunha, a maior proporção de agregados foi para os fisiogênicos, em ambas as profundidades (Tabela 3). Esse padrão indica que o uso de dejetos suínos (DLS e CSS) favorece a via de formação de agregados biogênica em comparação a via fisiogênica, seja diretamente pela adição de matéria orgânica proveniente dos dejetos ou indiretamente pela maior exploração do solo via sistema radicular, pois a adição dos dejetos aumentou a produção de matéria seca da aveia preta (Tabela 2).

Entre os tratamentos com dejetos, na profundidade de $0-5 \mathrm{~cm}$, não foram observadas diferenças entre as proporções de agregados biogênicos e fisiogênicos. Para a profundidade de 5-10 cm, o tratamento com DLS2X apresentou a maior porcentagem de agregados biogênicos em relação aos demais. Isso pode estar relacionado com o tipo de material, que por ser líquido, penetra mais facilmente no solo até a profundidade de 5-10 cm, favorecendo a formação de agregados biogênicos, ao passo que a CSS, por ser sólida, fica mais limitada à superfície do solo.

Tabela 3. Massa (g) e porcentagem (\%) de agregados biogênicos e fisiogênicos nos diferentes sistemas de uso do solo em Braço do Norte, Santa Catarina, Brasil.

\begin{tabular}{lllcccc}
\hline \multicolumn{1}{c}{ Tratamentos } & $\begin{array}{l}\text { Prof. } \\
(\mathbf{c m})\end{array}$ & ${ }^{1} \mathbf{M A T}$ & MAB & MAF & ${ }^{2} \% \mathbf{A B}$ & \%AF \\
\hline \multirow{2}{*}{ Test } & $0-5$ & 906,32 & 297,87 & 608,45 & $33 \mathrm{Bb}$ & $67 \mathrm{Aa}$ \\
& $5-10$ & 981,04 & 184,15 & 797,25 & $19 \mathrm{Cb}$ & $81 \mathrm{Aa}$ \\
\hline \multirow{2}{*}{ DLS1X } & $0-5$ & 708,15 & 670,25 & 37,9 & $95 \mathrm{Aa}$ & $5 \mathrm{Bb}$ \\
& $5-10$ & 723,86 & 429,55 & 294,30 & $59 \mathrm{Ba}$ & $41 \mathrm{Bb}$ \\
\hline \multirow{2}{*}{ DLS2X } & $0-5$ & 1178,8 & 1099,90 & 78,90 & $93 \mathrm{Aa}$ & $7 \mathrm{Bb}$ \\
& $5-10$ & 1395,15 & 1242,05 & 153,10 & $89 \mathrm{Aa}$ & $11 \mathrm{Db}$ \\
\multirow{2}{*}{ CSS1X } & $0-5$ & 669,80 & 624,90 & 44,93 & $93 \mathrm{Aa}$ & $7 \mathrm{Bb}$ \\
& $5-10$ & 919,18 & 509,73 & 399,45 & $55 \mathrm{Ba}$ & $43 \mathrm{Bb}$ \\
\hline \multirow{2}{*}{ CSS2X } & $0-5$ & 736,1 & 669,0 & 67,3 & $91 \mathrm{Aa}$ & $9 \mathrm{Bb}$ \\
& $5-10$ & 776,2 & 511,0 & 265,2 & $66 \mathrm{Ba}$ & $34 \mathrm{Cb}$ \\
\hline
\end{tabular}

${ }^{1}$ Compreende a massa de agregados total do solo antes da quantificação, conforme a sua origem, em agregados biogênicos e fisiogênicos. ${ }^{2}$ Compreende a porcentagem de agregados biogênicos e fisiogênicos relacionadas à massa total de agregados do solo após a sua separação em biogênicos e fisiogênicos. Médias seguidas pela mesma letra, em cada profundidade, maiúscula na coluna não diferem entre os tratamentos para cada tipo de agregado e mesma letra minúscula na linha não difere entre os tipos de agregados para cada tratamento avaliado (Scott-Knott, $\mathrm{p}<0,05$ ). Test = testemunha (sem adubação com dejetos); DLS1X = dejeto líquido de suínos, 1 vez a recomendação de N; DLS2X = dejeto líquido de suínos, 2 vezes a recomendação de $\mathrm{N}$; CSS1X= cama sobreposta de suínos, 1 vez a recomendação de $\mathrm{N}$; CSS2X = cama sobreposta de suínos, 2 vezes a recomendação de N. MAT= massa de agregados total do solo; $\mathrm{MAB}=$ massa de agregados biogênicos; MAF= massa de agregados fisiogênicos; $\mathrm{AB}=$ agregados biogênicos; $\mathrm{AF}=$ agregados fisiogênicos. 
Loss et al. (2014) avaliando diferentes sistemas de uso do solo em Marmeleiro -PR, sendo eles floresta secundária, pastagem, SPD e SPC constataram que em todos os sistemas prevaleciam agregados fisiogênicos. Contudo, entre os sistemas avaliados, a pastagem apresentou maiores porcentagens de agregados biogênicos e o SPD se assemelhou a floresta secundária, sendo que no SPC prevaleceu $100 \%$ de agregados fisiogênicos. Os resultados encontrados foram atribuídos ao fato de o SPD, com ausência de revolvimento e manutenção da resteva do azevém, bem como a maior adição de carbono via sistema radicular da gramínea no sistema de uso do solo com pastagem, propicia melhores condições à fauna do solo, que são os principais expoentes na formação de agregados biogênicos (Batista et al., 2013). Além disso, também foram encontrados maiores teores de nutrientes e carbono nos agregados biogênicos em comparação aos fisiogênicos (Loss et al., 2014).

Dessa forma, informações sobre o estado da agregação da camada superficial do solo constituem-se em indicadores de qualidade relativamente simples e sensíveis às mudanças decorrentes da adição de dejetos de suínos ao solo como via de fornecimento de nutrientes para as plantas. 0 aumento da atividade biológica nas áreas com adição de dejetos suínos (Morales et al. 2016) resultou em alterações nas diferentes vias de formação de agregados, tais como a maior porcentagem de agregados biogênicos em relação aos fisiogênicos. A manutenção da palhada vegetal sobre o solo, a ausência de revolvimento do mesmo e a adição dos dejetos de suínos acarretam em melhores condições físicas e químicas, com posterioraumento da fauna do solo, principalmente minhocas (Anghinoni et al., 2011). Assim, têm-se a formação dos agregados biogênicos, pois estes estão diretamente relacionados com a fauna do solo (Batista et al., 2013).

Diâmetro médio ponderado dos agregados. Não foram verificadas diferenças para DMP na profundidade de $0-5 \mathrm{~cm}$ entre os diferentes tratamentos e entre os tipos de agregados. $\mathrm{Na}$ profundidade de 5-10 cm, o uso de dejetos suínos aumentou o DMP em relação ao tratamento testemunha, com ênfase para o uso de CSS1X e CSS2X para agregados biogênicos e DLS e CSS para agregados fisiogênicos (Tabela 4).

A ausência de diferenças para DMP na camada superficial do solo $(0-5 \mathrm{~cm})$ indica que o SPD com a sucessão aveia preta/milho é eficiente para manter os elevados índices de agregação do solo $(4,550$ a $4,723 \mathrm{~mm}$, respectivamente para agregados biogênicos e fisiogênicos na área testemunha), mesmo sem a adição de matéria orgânica via dejetos de suínos (DLS e CSS). Isso é decorrente da cobertura do solo proporcionada pelo milho e especialmente pela aveia, pois as gramíneas possuem alta capacidade de regeneração, produção de matéria seca (Tabela 2) e densidade radicular, o que favorece a agregação do solo, pelo efeito que exercem na aglutinação de suas partículas e na estabilização dos agregados resultantes (Tivet et al., 2013).

Na profundidade de 5-10 cm, o uso dos dejetos de suínos (DLS e CSS) acarretou na maior disponibilidade de nutrientes e carbono orgânico total (COT) (Loss et al., 2017) no solo. Assim, têm-se melhores condições químicas para 0 desenvolvimento da aveia preta, favorecendo a produção de matéria seca (Tabela 2), e maior desenvolvimento radicular, o que aumenta a agregação do solo, pelo efeito que o sistema radicularexercenaaproximação desuaspartículas, com posterior aumento na estabilização dos agregados resultantes, acarretando em maiores valores de DMP. Segundo Loss et al. (2017), os teores de COT variaram de 27,00 a 63,20 $\mathrm{g} \mathrm{kg}^{-1}$ nos agregados biogênicos e de 21,20 a 51,90 $\mathrm{g} \mathrm{kg}^{-1}$ nos fisiogênicos. Os maiores teores de COT foram encontrados nos tratamentos com CSS1X (46,59 e 37,47 $\mathrm{g} \mathrm{kg}^{-1}$ ) e CSS2X (53,58 e 44,09 $\mathrm{g} \mathrm{kg}^{-1}$ ) para os agregados biogênicos e fisiogênicos, respectivamente. A testemunha, DLS1X e DLS2X apresentaram os teores de 33,11 e 28,58 $\mathrm{g} \mathrm{kg}^{-1}$; 37,15 e 28,33 $\mathrm{g} \mathrm{kg}^{-1}$; 37,19 e 27,09 $\mathrm{g} \mathrm{kg}^{-1}$, respectivamente, para os agregados biogênicos e fisiogênicos. 
Tabela 4. Diâmetro médio ponderado (DMP, mm) de agregados biogênicos e fisiogênicos.

\begin{tabular}{lcccc}
\hline \multirow{2}{*}{ Tratamentos } & \multicolumn{1}{c}{ Biogênico } & Fisiogênico & Biogênico & Fisiogênico \\
\cline { 2 - 5 } & \multicolumn{2}{c}{$\mathbf{0 - 5} \mathbf{~ c m}$} & \multicolumn{2}{c}{$\mathbf{5 - 1 0} \mathbf{~ c m}$} \\
\hline Testemunha & $4,550 \mathrm{Aa}$ & $4,723 \mathrm{Aa}$ & $4,245 \mathrm{Ba}$ & $4,192 \mathrm{Ca}$ \\
DLS1X & $4,704 \mathrm{Aa}$ & $4,832 \mathrm{Aa}$ & $4,465 \mathrm{Ba}$ & $4,606 \mathrm{Aa}$ \\
DLS2X & $4,432 \mathrm{Aa}$ & $4,718 \mathrm{Aa}$ & $4,261 \mathrm{Ba}$ & $4,461 \mathrm{Ba}$ \\
CSS1X & $4,736 \mathrm{Aa}$ & $4,843 \mathrm{Aa}$ & $4,895 \mathrm{Aa}$ & $4,852 \mathrm{Aa}$ \\
CSS2X & $4,641 \mathrm{Aa}$ & $4,813 \mathrm{Aa}$ & $4,853 \mathrm{Aa}$ & $4,591 \mathrm{Ab}$ \\
CV(\%) & 3,94 & 3,34 & 4,67 & 4,11 \\
\hline
\end{tabular}

Médias seguidas pela mesma letra maiúscula na coluna não diferem entre os sistemas de uso do solo para cada tipo de agregado e mesma letra minúscula na linha não difere entre os tipos de agregados para cada sistema avaliado (teste Scott-Knott, $\mathrm{p}<0,05$ ). Testemunha = sem adubação com dejetos; DLS1X = dejeto líquido de suínos, 1 vez a recomendação de N; DLS2X = dejeto líquido de suínos, 2 vezes a recomendação de N; CSS1X= cama sobreposta de suínos, 1 vez a recomendação de N; CSS2X = cama sobreposta de suínos, 2 vezes a recomendação de $\mathrm{N}$. CV = coeficiente de variação.

Nos agregados biogênicos, apenas as áreas com CSS diferiram das demais, apresentando maiores valores de DMP. Entretanto, nos agregados fisiogênicos verificaram-se maiores variações entre os tratamentos, com menores valores de DMP para a área testemunha, valores intermediários para DLS2X e maiores índices de DMP para as áreas com DLS1X e CSS (Tabela 4). Estas diferenças podem ser decorrentes da absorção de água continuamente pelas raízes, promovendo uma secagem local e contribuindo para aumentar a coesão entre as partículas sólidas do solo (Brandâo \& Silva, 2012), favorecendo os agregados fisiogênicos, que são formados por ciclos de umedecimento esecagem. As gramíneas contribuem nos processos de formação e estabilização de agregados do solo, devido, principalmente, à alta densidade de suas raízes, que promove a aproximação de partículas pela constante absorção de água do solo, como também às periódicas renovações do sistema radicular e à uniforme distribuição dos exsudados orgânicos ao solo, que estimulam a atividade microbiana, cujos subprodutos também atuam na estabilização dos agregados (Brandâo \& Silva, 2012).
Nos agregados biogênicos, os maiores teores de COT nos tratamentos com CSS $(46,59$ e 37,47g $\mathrm{kg}^{-1}$, respectivamente para os biogênicos e fisiogênicos em CSS1X; e 53,58 e 44,09 $\mathrm{g} \mathrm{kg}^{-1}$, respectivamente para os biogênicos e fisiogênicos em CSS2X; segundo Loss et al. (2017) macroagregados mais estáveis em água (Tabela 5), consequentemente, com maiores índices de DMP. Esses maiores teores nos tratamentos com CSS também podem favorecer a retenção de maiores conteúdos de umidade do solo, acarretando em melhores condições para a biota do solo e desenvolvimento radicular, que são fatores primordiais para formação dos agregados biogênicos. A ausência de diferenças entre a área testemunha e DLS pode ser devido à baixa quantidade de MS (Tabela 1) e, consequentemente, baixa relação C/N dos DLS quando comparada aos altos teores de MS e alta relação $\mathrm{C} / \mathrm{N}$ dos dejetos de CSS (Tabela 1). As maiores quantidades de MS e maior relação $\mathrm{C} / \mathrm{N}$ favorece uma decomposição mais lenta dos materiais orgânicos, acarretando em maiores teores de COT (Loss et al., 2017) e, consequentemente, maior quantidade de agente cimentante para favorecer o processo de formação e estabilização dos agregados do solo. 
Tabela 5. Distribuição da massa (g) de agregados biogênicos e fisiogênicos em diferentes sistemas de uso do solo em Braço do Norte, SC.

\begin{tabular}{|c|c|c|c|c|c|c|}
\hline \multirow{3}{*}{ Tratamentos } & \multicolumn{2}{|c|}{$\begin{array}{l}\text { Macroagregados } \\
(8,0>X \geq 2,0 \mathrm{~mm})\end{array}$} & \multicolumn{2}{|c|}{$\begin{array}{c}\text { Mesoagregados } \\
(2,0>X \geq 0,25 \mathrm{~mm}\end{array}$} & \multicolumn{2}{|c|}{$\begin{array}{c}\text { Microagregados } \\
(0,25>X \geq 0,105 \mathrm{~mm})\end{array}$} \\
\hline & Biogênico & Fisiogênico & Biogênico & Fisiogênico & Biogênico & Fisiogênico \\
\hline & \multicolumn{6}{|c|}{$0-5 \mathrm{~cm}$} \\
\hline Test & $21,64 \mathrm{Aa}$ & $22,40 \mathrm{Aa}$ & $1,83 \mathrm{Aa}$ & 1,73 Aa & $1,51 \mathrm{Aa}$ & 0,34 Bb \\
\hline DLS1X & $22,74 \mathrm{Aa}$ & $23,75 \mathrm{Aa}$ & $0,91 \mathrm{Ba}$ & $0,71 \mathrm{Ca}$ & 0,86 Ba & $0,35 \mathrm{Bb}$ \\
\hline DLS2X & $21,25 \mathrm{Aa}$ & $22,70 \mathrm{Aa}$ & $1,58 \mathrm{Aa}$ & $1,00 \mathrm{Bb}$ & $1,59 \mathrm{Aa}$ & $0,49 \mathrm{Ab}$ \\
\hline CSS1X & $22,97 \mathrm{Aa}$ & $23,77 \mathrm{Aa}$ & $0,61 \mathrm{Ca}$ & $0,53 \mathrm{Ca}$ & 0,94 Ba & 0,33 Bb \\
\hline $\operatorname{css} 2 \mathrm{X}$ & $21,19 \mathrm{Aa}$ & $22,49 \mathrm{Aa}$ & $1,08 \mathrm{Ba}$ & $0,46 \mathrm{Cb}$ & 0,91 Ba & $0,51 \mathrm{Ab}$ \\
\hline \multirow[t]{2}{*}{ CV $(\%)$} & 3,85 & 3,76 & 14,07 & 22,27 & 16,26 & 12,01 \\
\hline & \multicolumn{6}{|c|}{$5-10 \mathrm{~cm}$} \\
\hline Test & $21,11 \mathrm{Ba}$ & $20,04 \mathrm{Ba}$ & $1,46 \mathrm{Bb}$ & $3,05 \mathrm{Aa}$ & $2,19 \mathrm{Aa}$ & $1,56 \mathrm{Ab}$ \\
\hline DLS1X & $21,61 \mathrm{Ba}$ & $22,44 \mathrm{Aa}$ & 1,54 Ba & $1,55 \mathrm{Ca}$ & 1,63 Ba & 0,64 Bb \\
\hline DLS2X & $21,17 \mathrm{Ba}$ & $21,55 \mathrm{Ba}$ & 3,63 Aa & $2,24 \mathrm{Bb}$ & $0,76 \mathrm{Ca}$ & 0,80 Ba \\
\hline CSS1X & $23,82 \mathrm{Aa}$ & $23,71 \mathrm{Aa}$ & $0,39 \mathrm{Ca}$ & 0,59 Da & $0,29 \mathrm{Da}$ & 0,38 Ba \\
\hline $\operatorname{css} 2 \mathrm{X}$ & $23,78 \mathrm{Aa}$ & $22,31 \mathrm{Aa}$ & $0,31 \mathrm{Cb}$ & $1,47 \mathrm{Ba}$ & $0,43 \mathrm{Db}$ & 0,76 Ba \\
\hline CV(\%) & 4,80 & 4,08 & 15,23 & 16,84 & 9,01 & 23,30 \\
\hline
\end{tabular}

Médias seguidas pela mesma letra maiúscula na coluna não diferem entre os tratamentos para cada tipo de agregado e mesma letra minúscula na linha não difere entre os tipos de agregados para cada sistema avaliado (teste Scott-Knott, p <0,05). Test = testemunha (sem adubação com dejetos); DLS1X = dejeto líquido de suínos, 1 vez a recomendação de N; DLS2X = dejeto líquido de suínos, 2 vezes a recomendação de N; CSS1X= cama sobreposta de suínos, 1 vez a recomendação de N; CSS2X = cama sobreposta de suínos, 2 vezes a recomendação de N. CV = coeficiente de variação.

Distribuição dos agregados em macro, meso e microagregados. De maneira geral, verificouse predominância de macroagregados em relação aos meso e microagregados (Tabela 5). Para os macroagregados, não foram verificadas diferenças entre os tratamentos e os tipos de agregados, na profundidade de $0-5 \mathrm{~cm}$. Isto indica que a manutenção dos resíduos vegetais da aveia preta e do milho na superfície do solo em SPD tem maior importância na formação e manutenção de agregados de maior tamanho em comparação aos tratamentos com adição de dejetos suínos.

A ausência de diferenças entre os tratamentos em relação aos macroagregados na camada superficial do solo corrobora com a similaridade entre os valores de DMP encontrados nesta profundidade (Tabela 4). Para a camada de 5-10 cm, as áreas com CSS apresentaram maiores valores de macroagregados, tanto biogênicos quanto fisiogênicos, sendo nestes últimos verificados os maiores valores também para a área com DLS1X. Estes resultados corroboram os maiores valores de DMP encontrados nessas áreas (Tabela 4) e indica que o uso desses dejetos de suínos, em profundidade, favorece a formação de macroagregados, principalmente o uso de CSS1X ou CSS2X, devido aos maiores teores de MS, C/N e nutrientes, tais como o Ca e Mg (Tabela 1), assim como os maiores teores de COT (Loss et al., 2017) no solo adubado com CSS. 
Os maiores valores de macroagregados observados nos tratamentos com CSS também podem estar relacionados com a maior atividade microbiana encontrada nesses tratamentos quando comparado aos demais (Morales et al., 2016), resultando na formação de macroagregados poração deforças eletrostáticas, atividade microbiana e crescimento de raízes (Six et al., 2000). A maior quantidade de macroagregados nos tratamentos com CSS na profundidade de $5-10 \mathrm{~cm}$ acarreta em melhores condições físicas para o desenvolvimento radicular das culturas, sendo verificado nesta mesma área de estudo, segundo Comin et al. (2013), menores valores de densidade do solo e resistência a penetração, assim como maiores valores de macroporosidade nos tratamentos com CSS.

Para os meso e microagregados, de maneira geral, verificaram-se que as áreas com CSS apresentaram os menores valores, principalmente para os biogênicos. Isto indica que os macroagregados formados nas áreas com CSS são mais estáveis em água e não se rompem em agregados menores quando submetidos ao processo de estabilidade via úmida, principalmente se comparado com a área testemunha, que apresentou maiores valores de água.

As diferenças encontradas na distribuição dos agregados após peneiramento por via úmida podem ser decorrentes da qualidade do material vegetal (Spaccini et al. (2004). Resíduos de fácil decomposição acarretam em rápido aumento na agregação das partículas, entretanto, com efeito na estrutura do solo de caráter temporário. Em contrapartida, se o material que compõe o resíduo é de lenta decomposição, o efeito da agregação será menor, mas mais duradouro, o que sugere que o composto de resíduos orgânicos devem ser preparados com materiais ricos em lignina (Celik et al., 2004).
Dessa forma, nos tratamentos com CSS, por apresentarem maior quantidade de MS e maior relação $\mathrm{C} / \mathrm{N}$ que os DLS (Tabela 1 ), o efeito da agregação será mais prolongado, culminando em agregados mais estáveis e, consequentemente, menores massas de agregados de menor tamanho. Enquanto nos tratamentos com DLS, que apresentam menores quantidades de MS e menor relação $\mathrm{C} / \mathrm{N}$ em comparação à CSS (Tabela 1), o efeito na estrutura do solo será de caráter mais temporário. Porém, quando se compara o efeito do uso dos DLS com a área testemunha, principalmente na menor dose (DLS1X), verifica-se que tem-se valores menores ou iguais aos encontrados na área testemunha para meso e microagregados biogênicos e fisiogênicos (Tabela 5), indicando que o uso de DLS1X associado ao SPD aumenta o efeito da agregação do solo, apresentando macroagregados mais estáveis em água e, consequentemente, menores massas de meso e microagregados. Este padrão pode ser verificado na profundidade de 5-10 cm (Tabela 5), na qual a área com DLS1X apresentou maiores valores de macroagregados fisiogênicos e menores valores para mesoagregados (fisiogênicos) e microagregados (biogênicos e fisiogênicos) em comparação à área testemunha.

As profundidades avaliadas neste estudo (0-5 e 5-10 cm) fazem parte do horizonte A do solo (Argissolo Vermelho-Amarelo substrato granito, textura média), que apresenta altos teores de COT (Loss et al., 2017), e consequentemente apresenta baixos conteúdos de óxidos de ferro e alumínio. Assim, nos processos relacionados à agregação do solo tem-se maior importância da matéria orgânica quando comparado aos óxidos, indicando que nessa camada de 0-10 cm, a agregação do solo segue a teoria da hierarquia dos agregados, onde agregados menores se unem, formando os agregados maiores (Tivet et al., 2013). Dessa forma, tem-se a interação da argila com cátions polivalentes e compostos orgânicos persistentes, tais como o carbono da 
fração humina (Ventura et al., 2018), favorecendo a formação dos microagregados. Por sua vez, da união dos microagregados surgem os macroagregados, que são estabilizados por meio de compostos transitórios, tais como a matéria orgânica particulada, adicionada ao solo via sistema radicular e deposição de resíduos vegetais.

Entre os tipos de agregados, verificaram-se diferenças apenas para meso e microagregados, commaioresvalorespara os biogênicos(Tabela5). Estes resultados são decorrentes dos maiores teores de carbono orgânico particulado (COp) encontrado nos agregados biogênicos (Ventura et al., 2018). 0 COp (ou a matéria orgânica particulada) pode atuar como pontes de nucleação na formação de microagregados no interior de macroagregados (Golchin et al., 1994). Com o consumo do COp, decresce a atividade microbiana e a liberação de mucilagens e o macroagregado perde a estabilidade, eventualmente ocorrendo o seu rompimento e liberando os microagregados (Tivet et al., 2013).

\section{CONCLUSÕES}

A adição de dejetos suínos aumentou a formação de agregados biogênicos. Na área testemunha prevalece a via de formação de agregados fisiogênicos. A aplicação de DLS não alterou o DMP e a distribuição dos macroagregados em relação à testemunha, enquanto a aplicação de CSS aumentou esses parâmetros quando comparada aos tratamentos DLS e Testemunha.

\section{AGRADECIMENTOS}

Ao Conselho Nacional de Desenvolvimento Científico e Tecnológico (CNPq) pelo auxílio financeiro por meio da Chamada Universal Projeto n. 471345/2012-1. E também à Fundação Agrisus pelo auxílio financeiro decorrente do projeto PA 1087-13.

Conflito de interesses: Os autores declaram não haver conflito de interesse.

\section{REFERÊNCIAS BIBLIOGRÁFICAS}

Anghinoni, I., Moraes, A., Carvalho, P. C. F., Souza, E. D., Conte, O. \& Lang, C. R. (2011). Benefícios da integração lavoura-pecuária sobre a fertilidade do solo em sistema plantio direto. In: Fonseca, A. F., Caires, E. F., Barth, G. Fertilidade do solo e nutrição de plantas no sistema plantio direto. pp. 1-31. Ponta Grosa: AEACG/Inpag.

Batista, I., Fernandes, M., Pereira, M., Bieluczyk, W., Schiavo, J. \& Mello, N. (2013). Caracterização dos agregados em solos sob cultivo no Cerrado, MS. Semina: Ciências Agrárias. 34(4): 1535-1548. doi: 10.5433/1679-0359.2013v34n4p1535

Brandâo, E. \& Silva, I. (2012). Formação e estabilização de agregados pelo sistema radicular de braquiária em um Nitossolo Vermelho. Ciência Rural. 42(7): 1193-1199. doi: 10.1590/S010384782012000700009.

Celik, I., Ortas, I. \& Kilic, S. (2004). Effects of compost, mycorrhiza, manure and fertilizer on some physical properties of a Chromoxerert soil. Soil Tillage Research, 78(1): 59-67. doi: 10.1016/j. still.2004.02.012

Comin, J., Loss, A., Veiga, M., Guardini, R., Schmitt, D., Victoria Oliveira, P., Filho, P., Couto, R., Benedet, L., Júnior, V. \& Brunetto, G. (2013). Physical properties and organic carbon content of a Typic Hapludult soil fertilised with pig slurry and pig litter in a no-tillage system. Soil Research, 51(5): 459-470. doi: 10.1071/SR13130

CFSRS/SC - Comissão de Química e Fertilidade do Solo. (1995). Manual de adubação e calagem para os estados do Rio Grande do Sul e Santa Catarina. 9. ed. Porto Alegre: Sociedade Brasileira de Ciência do Solo.

CQFSRS/SC - Comissão de Química e Fertilidade do Solo. (2004). Manual de adubação e calagem para os estados do Rio Grande do Sul e Santa Catarina. 10. ed. Porto Alegre: Núcleo Regional Sul Sociedade Brasileira de Ciência do Solo. Segunda reimpressão. 400p. 
Embrapa - Empresa Brasileira de Pesquisa Agropecuária. (1997). Manual de métodos de análise de solos, Rio de Janeiro: EMBRAPA-CNPS. 212p.

Embrapa - Empresa Brasileira de Pesquisa Agropecuária. (2013). Sistema Brasileiro de Classificação de Solos. 3aed. Brasília: Rio de Janeiro: Embrapa Solos. 312p.

Fernandes, J., Pereira, M., Silva Neto, E. \& Correa, T. (2017).Characterization of biogenic, intermediate and physicogenic soil aggregates of areas in the brazilian atlantic forest. Revista Caatinga, 30(1): 59-67. doi: 10.1590/1983-21252017v30n107rc

Giacomini, S. \& Aita, C. (2008). Cama sobreposta e dejetos líquidos de suínos como fonte de nitrogênio ao milho. Revista Brasileira de Ciência do Solo, 32(1): 195-205. doi: 10.1590/S010006832008000100019

Golchin, A., Oades, J., Skjemstad, J. \& Clarke, P. (1994). Study of free and occluded particulate organic matter in soils by solid state 13C CP/MAS NMR spectroscopy and scanning electron microscopy. Australian Journal of Soil Research, 32(2): 285309. doi: 10.1071/SR9940285

IBGE - Instituto Brasileiro de Geografia e Estatística. (2016). Pesquisa Pecuária Municipal. Recuperado de https://www.ibge. gov.br/estatisticas-novoportal/economicas/ agricultura-e-pecuaria/9107-producao-dapecuaria-municipal.html?\&t=resultados.

Jouquet, P., Zangerle, A., RumpeL, C., Brunet, D., Bottinelli, N. \& Tranduc, T. (2009). Relevance and limitations of biogenic and physicogenic classification: a comparison of approaches for differentiating the origin of soil aggregates. European Journal of Soil Science. 60(6): 11171125. doi: 10.1111/j.1365-2389.2009.01168.x

Kampf, N. \& Curi, N. (2012). Caracterização do Solo. In: Pedologia: Fundamentos/ Joao Carlos Ker (ed). Viçosa: Sociedade Brasileira de Ciência do Solo. 343p.

Loss, A., Pereira, M., Costa, E. \& Beutler, S. (2014). Soil fertility, physical and chemical organic matter fractions, natural $13 \mathrm{C}$ and $15 \mathrm{~N}$ abundance in biogenic and physicogenic aggregates in areas under different land use systems. Soil Research. 52(7): 685-697. doi: 10.1071/SR14045

Loss, A., Lourenzi, C. R., Mergen Junior, C. A., Santos Junior, E., Benedet, L., Pereira, M., Piccolo, M. C., Brunetto, G., Lovato, P. \& Comin, J. (2017). Carbon, nitrogen and natural abundance of $13 \mathrm{C}$ and $15 \mathrm{~N}$ in biogenic and physicogenic aggregates in a soil with 10 years of pig manure application. Soil \& Tillage Research, 166:52-58. doi: 10.1016/j. still.2016.10.007

Morales, D., Vargas, M., Oliveira, M., Taffe, B., Comin, J., Soares, C. \& Lovato, P. (2016). Response of soil microbiota to nine-year application of swine manure and urea. Ciência Rural, 46(2):260-266. doi: 10.1590/0103-8478cr20140565

Seganfredo, M. (2007). Uso de dejetos suínos como fertilizantes e seus riscos ambientais. Brasília, DF: Embrapa Informação Tecnológica. 302p.

Silva, C., Pereira, M. \& Fernandes, J. (2016). Correa NETO, T.A. Aggregate formation and soil organic matter under different vegetation types in Atlantic Forest from Southeastern Brazil. Semina: Ciências Agrárias. 37(6): 3927-3940. doi: 10.5433/1679-0359.2016v37n6p3927

Silva, L., Silva, I., Inda J. \& Nascimento, P. (2010). Atributos físicos e químicos de agregados pedogênicos e de coprólitos de minhocas em diferentes classes de solos da Paraíba. Ciência e Agrotecnolgia. 34(6): 1365-1371. doi: 10.1590/ S1413-70542010000600002.

Spaccini, R., Mbagwub, J.S.C., Igweb, C.A. Conte, P. Piccolo, A. (2004). Carbohydrates and aggregation in lowland soils of Nigeria as influenced by organic inputs. Soil and Tillage Research, 75:161172. doi: https://doi.org/10.1016/S01671987(03)00158-2

Six, J., Elliott, E. \& Paustian, K. (2000). Soil macroaggregate turnover and microaggregate formation: a mechanism for $\mathrm{C}$ sequestration under no-tillage agriculture. Soil Biology \& Biochemestry. 32(14): 2099-2103. doi: https:// doi.org/10.1016/S0038-0717(00)00179-6 
Tivet, F.; Sá, J.C.M.; Lal, R.; Briedis, C.; Borszowskei, P.R.; Santos, J.B.; Farias, A.; Eurich, G.; Hartman, D.C.; Nadolny Junior, M.; Bouzinac, S.; Séguy, L. (2013). Aggregate $C$ depletion by plowing and its restoration by diverse biomass- $\mathrm{C}$ inputs under no-till in sub-tropical and tropical regions of Brazil. Soil and Tillage Research, 126:203-218. doi: https://doi.org/10.1016/j.still.2012.09.004

Velasquez, E., Pelosi, C., Brunet, D., Grimaldi, M., Martins, M., Rendeiro, A. C., Barrios, E. \& Lavelle, P. (2007). This ped is my ped: Visual separation and near infrared spectra allow determination of the origins of soil macroaggregates. Pedobiologia. 51(1):75-87. doi: https://doi.org/10.1016/j. pedobi.2007.01.002

Ventura, B., Loss, A., Giumbelli, L., Ferreira, G., Bueno, A., Lourenzi, C., Brunetto, G. \& Comin, J. (2018). Carbon, nitrogen and humic substances in biogenic and physicogenic aggregates of a soil with a 10-year history of successive applications of swine waste. Tropical and Subtropical Agroecosystems. 21(2): 329-343.

Vezzani, F. M. \& Mielniczuk, J. (2011). Agregação e estoque de carbono em argissolo submetido a diferentes práticas de manejo agrícola. Revista Brasileira de Ciência do Solo. 35(1): 213223. doi: http://dx.doi.org/10.1590/S010006832011000100020

Yague, M.R., Bosch-Serra, A.D., Antùnez, M. \& Boixadera, J. (2012). Pig slurry and mineral fertilization strategies' effects on soil quality: Macroaggregate stability and organic matter fractions. Science of the Total Environment. 438: 218-224. doi: 10.1016/j.scitotenv.2012.08.063 